\title{
РАЗРАБОТКА МАТЕМАТИЧЕСКОЙ МОДЕЛИ ПРОЦЕССА ДЕСУБЛИМАЦИИ И ЕЁ АПРОБАЦИЯ ДЛЯ МНОГОКАМЕРНОГО АППАРАТА
}

\author{
И. Ю. Русаков, В. Л. Софрронов, В.Н.Брендаков
}

В настоящей работе проведены изыскания по разработке конструкции ядернобезопасного десублиматора для конденсации паров высокообогащённого гексафрторида урана из парогазовой смеси и изучение процесса десублимации этого продукта в новой предложенной конструкции аппарата на математической модели. Разработанный десублиматор представляет собой вертикальный кольцевой аппарат с обогреваемыми стенками, внутренняя полость которого разделена поперечными перегородками на ряд последовательно сообщающихся камер. Разработанные авторами фризическая и математическая модели для процесса десублимации в предложенном десублиматоре рассматривают сопряжённую задачу гидродинамики и теплообмена, решение которой позволяет при заданных граничных условиях рассчитывать картины линий тока парогазовой смеси и поля температур в каждой из камер десублиматора. Результатом расчёта является десублиматор, исключающий потери целевого продукта в виде аэрозолей, тем более недопустимые для радиационноопасных веществ, к которым относится гексафоторид урана.

Ключевые слова: гексафрторид урана, поверхностная десублимация, объёмная десублимация, десублиматор, фризическая модель, математическая модель.

\section{ВВЕДЕНИЕ}

Процессы сублимации и десублимации (конденсации) веществ используются для разделения и сбора целевых продуктов [1-3]. Эти процессы разделяются на процессы, протекающие на поверхности стенок и в объёме. В реальных аппаратах оба процесса протекают одновременно, но в зависимости от конструкции аппарата и режима работы оборудования основным видом десублимации является только один из перечисленных, а второй - только сопутствующим. При этом сопутствующий процесс десублимации обычно ухудшает показатели основного типа десублимации и, как следствие, всего процесса в целом.

При поверхностной десублимации в качестве основной целевой продукт в основной массе десублимируется на охлаждаемой поверхности, а часть продукта конденсируется в объёме аппарата в виде аэрозолей в количестве, пропорциональном парциальному давлению этого продукта [3]. Суммарное количество аэрозолей и газовой фазы определяет проскок рассматриваемого продукта через аппарат, т. е. качество его улавливания.

Существует большое количество разновидностей аппаратов-конденсаторов [2, 4-7], выбор и применение которых определяется масштабом и особенностями производства и характеристиками целевого продукта и пароПОЛЗУНОВСКИЙ ВЕСТНИК № 42018 газовой смеси (ПГС) в целом. Наиболее простыми типами десублиматора являются аппараты с цилиндрической охлаждаемой стенкой и с трубками Фильда, с гофррированной и оребрённой теплообменной поверхностью.

В атомной промышленности наиболее распространён способ поверхностной десублимации при извлечении гексафторида урана (ГФУ) из ПГС, в которой кроме ГФУ присутствуют другие летучие продукты реакции: кислород, фртороводород, фрториды и оксифрториды примесных элементов (Pu, F, N, V, $\mathrm{Mo}, \mathrm{W}, \mathrm{Cr}, \mathrm{B}, \mathrm{Si}, \mathrm{P})$. Потери целевого продукта в виде аэрозолей тем более недопустимы для радиационно-опасных веществ, к которым относится гексафторид урана. Исследования десублимации на реальном продукте является задачей достаточно трудоёмкой ввиду специфики производства и самого ГФУ, поэтому дать оценку эффрективности работы десублиматора рациональнее посредством математического моделирования.

\section{РАЗРАБОТКА КОНСТРУКЦИИ ДЕСУБЛИМАТОРА}

Наиболее эфффективным способом десублимации является такой, при котором в условиях изменяющегося режима десублимации из-за нарастания слоя десублимата на 
охлаждаемой стенке удалось бы избежать процесса появления аэрозолей и контролировать образование и толщину слоя десублимата, обеспечивая максимальное заполнение аппарата.

Для улавливания продукта, выделенного в объёме, можно использовать циклоны, скрубберы или фильтры, а для улавливания недесублимированных паров - дополнительные (контрольные) десублиматоры. Однако в условиях атомного производства технологически возможно использовать только контрольный десублиматор, который устанавливают после «основного» десублиматора, из которого продукт периодически извлекают для дальнейшей переработки, и он не относится к потерям.

С учётом имеющегося опыта нами было предложено «объединить» основной и контрольные десублиматоры в одном корпусе аппарата. Таким образом появилась конструкция многокамерного десублиматора с «тёплой» стенкой [8], схема которого показана на рисунке 1.

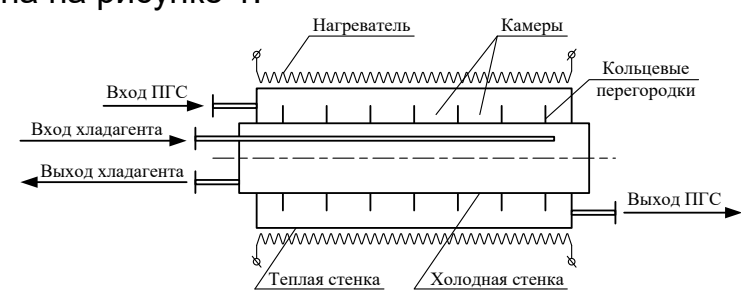

Рисунок 1 - Схема многокамерного десублиматора с «тёплой» стенкой

В таком аппарате десублимация целевого продукта будет происходить преимущественно на холодной цилиндрической стенке между кольцевыми перегородками в нескольких последовательно расположенных кольцевых камерах, где формируется динамический фронт десублимации. По мере заполнения первых камер фронт перемещается к выходу из аппарата (рисунок 2). При этом механизм заполнения камер десублиматора следующий. В начальный момент времени десублимация продукта происходит преимущественно в первой камере (линия …...........). По мере заполнения камеры I начинается образование слоя десублимата в камере II (линия - - - ). Причем в этот период в камере II слой формируется за счёт проскока аэрозолей из камеры I, то есть камера II при этом выполняет функцию контрольной. При дальнейшем протекании процесса появляется десублимат и в камере III (линия- . - - ).

Десублиматор предложенной конструкции обладает рядом преимуществ:

- обеспечивает постоянную производительность на период заполнения всех камер, за исключением двух последних (контрольных);

- минимизирует аэрозольный проскок целевого продукта из аппарата;

- гарантирует прохождение парогазовой смеси вдоль обогреваемой стенки;

- позволяет снизить затраты хладагента на десублимацию за счёт исключения потерь холода в окружающую среду.

\section{РАЗРАБОТКА МАТЕМАТИЧЕСКОЙ МОДЕЛИ И ЕЁ ОПРОБАЦИЯ}

Во время работы аппарата в нём имеют место процессы теплообмена и массообмена (выделения твёрдой фразы), ламинарного и турбулентно-ламинарного (в разных зонах) течения газовой среды переменной массы, нарастание слоя десублимата на стенке, изменение теплофизических параметров теплообмена внутри секций и условий десублимации, а также заполнение аппарата продуктом.

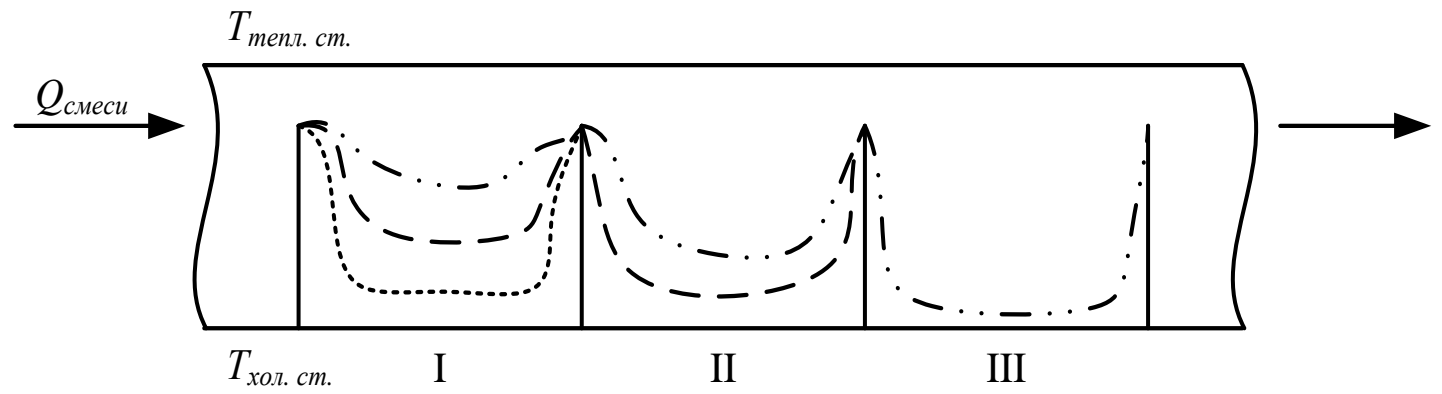

линия .............. - заполнение камеры / в начальный момент времени десублимации;

линия - - - - - образование слоя десублимата в камере II по мере заполнения камеры I; линия - . - - последующее заполнение камеры III

Рисунок 2 - Распределение десублимата по камерам десублиматора 


\section{РАЗРАБОТКА МАТЕМАТИЧЕСКОЙ МОДЕЛИ ПРОЦЕССА ДЕСУБЛИМАЦИИ И ЕЁ АПРОБАЦИЯ ДЛЯ МНОГОКАМЕРНОГО АППАРАТА}

По мере увеличения толщины слоя продукта в секциях изменяется соотношение поверхностной и объёмной десублимаций.

Каждый из перечисленных процессов имеет описание в технической литературе $[3,9]$, однако их полное комплексное описание и совместное рассмотрение представляется довольно сложным и трудоёмким. Учитывая, что многокамерный десублиматор представляет собой набор последовательно расположенных камер, в каждой из которых происходит поверхностная десублимация, а образующиеся аэрозоли повторно сублимируются и их пары конденсируются в следующих по ходу тока камерах, можно ограничиться только рассмотрением сопряжённой тепловой и гидродинамической задач в одной камере десублиматора. Решение задачи возможно для заданных радиальных размеров десублиматора $D_{0}$, D и длине камеры L (рисунок 3).

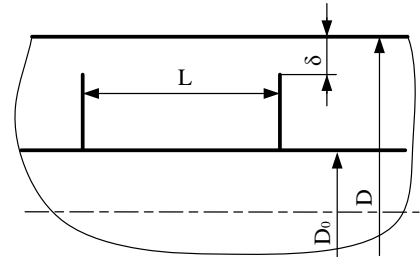

Рисунок 3 - Схема камеры десублиматора

Содержание ГФУ в ПГС в каждой камере пропорционально его парциальному давлению. С учетом этого возможно определить число камер, исключающее выгод ГФУ из аппарата.

Все физические процессы, происходящие в камере аппарата, описываются системой дифференциальных уравнений с частными производными, включающей уравнение неразрывности и уравнения Новье-Стокса [10]. Получить их решение можно только для тривиальных простых случаев течения. Поэтому для наших условий задачу решаем численно.

Для обезразмеривания уравнений выбираем характерные скорость и размер. В нашем случае это среднерасходная скорость $U_{0}$ во входном зазоре $\delta$ (рисунок 3) и приведённый диаметр Dп живого сечения кольца. В результате получаем безразмерный критерий, определяющий относительное значение вязких и инерционных эфффектов, называемый числом Рейнольдса $\mathrm{Re}=\mathrm{U}_{0} \cdot \mathrm{D}_{п} / \mathrm{v}$, где $\mathrm{v}-$ кинематическая вязкость ПГС. В случае осесимметричного течения удобно перейти к новым зависимым переменным «завихрённость $\Omega$ - функция тока $\psi »$, в результате чего из рассмотрения исключается градиент давле- ния. Этот искусственный математический приём позволяет существенно упростить схему расчёта.

Наличие в десублиматоре перегородок и узких зазоров для прохождения ПГС предполагает, даже несмотря на относительно малые скорости, развитое турбулентное течение. Моделирование турбулентности проведём известным способом. Для этого актуальные значения компонент скорости представим в виде $U=\mathrm{u}+\mathrm{u}^{\prime}$, где $\mathrm{u}$ - осреднённое по временному промежутку значение, u' - пульсационная составляющая. После подстановки таких значений и последующего осреднения по времени получаем уравнения, в которые кроме осреднённых значений зависимых переменных входят корреляции пульсаций компонент скорости. Эти корреляции и определяют природу дополнительных турбулентных напряжений, возникающих в потоке. Используя обобщённую гипотезу Буссинекса, введём понятие турбулентной вязкости. Существует большое число моделей турбулентности [11], однако для инженерных расчётов наиболее оптимальными по сложности и точности расчёта принято считать модели, в которых используется система из двух уравнений переносного типа, записанных для характеристик турбулентности. Исходя из этого, была выбрана следующая схема.

Расчёт гидродинамики в рабочем объёме камеры проводили на основе системы полных стационарных осесимметричных осреднённых дифференциальных уравнений Новье-Стокса эллиптического типа, записанных в переменных завихрённость $\Omega$, функция тока $\Psi$. Для замыкания системы уравнений

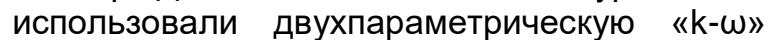
модель турбулентности Уилкокса [12]. Все уравнения записывали в цилиндрической системе координат, где $r, z$ - радиальная и аксиальная координаты, соответственно. Исходную систему уравнений в консервативном дивергентном и безвременном виде записали следующим образом:

- уравнение переноса завихрённости $\Omega$, полученное из уравнений движения путём исключения градиента давления

$$
\begin{aligned}
& \text { Re } \cdot r \cdot\left[u_{r} \cdot \frac{\partial \Omega}{\partial r}+u_{z} \cdot \frac{\partial \Omega}{\partial z}\right]-\frac{\partial}{\partial r}\left[r \cdot\left(1+v_{t}\right) \cdot \frac{\partial \Omega}{\partial r}\right]-\frac{\partial}{\partial z}\left[r \cdot\left(1+v_{t}\right) \cdot \frac{\partial \Omega}{\partial z}\right]= \\
& =\frac{\partial}{\partial r}\left\{\Omega \cdot \frac{\partial}{\partial r}\left[r \cdot\left(1+v_{t}\right)\right]\right\}+r \cdot \frac{\partial}{\partial z}\left(\Omega \cdot \frac{\partial v_{t}}{\partial z}\right)+2 \cdot \frac{\partial^{2} v_{t}}{\partial z^{2}} \cdot \frac{\partial u_{z}}{\partial r}+\frac{\partial}{\partial r}\left[\Omega \cdot\left(1+v_{t}\right)\right]+ \\
& +2 \cdot \frac{\partial^{2} v_{t}}{\partial r \partial z} \cdot\left(\frac{\partial u_{r}}{\partial r}-\frac{\partial u_{z}}{\partial z}\right)-2 \cdot \frac{\partial^{2} v_{t}}{\partial r^{2}} \cdot \frac{\partial u_{r}}{\partial z} ;
\end{aligned}
$$

- уравнение Пуассона для определения функции тока $\psi$, полученное из уравнения неразрывности 


$$
\frac{\partial^{2} \psi}{\partial r^{2}}+\frac{\partial^{2} \psi}{\partial z^{2}}=\Omega \cdot r^{2}+\frac{1}{r} \cdot \frac{\partial \psi}{\partial r}
$$

где

$$
\begin{aligned}
& \Omega=\frac{1}{r} \cdot\left(\frac{\partial u_{r}}{\partial z}-\frac{\partial u_{z}}{\partial r}\right), \\
& u_{r}=\frac{1}{r} \cdot \frac{\partial \psi}{\partial z}, \\
& u_{z}=-\frac{1}{r} \cdot \frac{\partial \psi}{\partial r}, \\
& v_{t}=\operatorname{Re} \cdot \frac{k}{\omega}
\end{aligned}
$$

- уравнение переноса кинетической энергии турбулентных пульсаций $k$.

$$
\begin{aligned}
& \text { Re } \cdot r \cdot\left[u_{r} \cdot \frac{\partial k}{\partial r}+u_{z} \cdot \frac{\partial k}{\partial z}\right]-\frac{\partial}{\partial r}\left[r \cdot\left(1+\sigma \cdot v_{t}\right) \frac{\partial k}{\partial r}\right]-\frac{\partial}{\partial z}\left[r \cdot\left(1+\sigma \cdot v_{t}\right) \frac{\partial k}{\partial z}\right]= \\
& =r \cdot G e n-\frac{2}{r} \cdot\left(\frac{2}{3} \cdot k+\frac{v_{t}}{\operatorname{Re}} \frac{\partial u_{z}}{\partial z}\right)-\operatorname{Re} \cdot r \cdot C_{\mu} \cdot k \cdot \omega
\end{aligned}
$$

где $k$ - кинетическая энергия турбулентных пульсаций;

$\omega$ - удельная скорость диссипации кинетической энергии турбулентных пульсаций;

- уравнение переноса удельной скорости диссипации $\omega$

$\operatorname{Re} \cdot r \cdot\left[u_{r} \frac{\partial \omega}{\partial r}+u_{z} \frac{\partial \omega}{\partial z}\right]-\frac{\partial}{\partial r}\left[r \cdot\left(1+\sigma \cdot v_{t}\right) \frac{\partial \omega}{\partial r}\right]-\frac{\partial}{\partial z}\left[r \cdot\left(1+\sigma \cdot v_{t}\right) \frac{\partial \omega}{\partial z}\right]=$

$=r \cdot \frac{\omega}{k} \cdot\left(C_{1} \cdot\right.$ Gen $\left.-C_{2} \cdot \operatorname{Re} \cdot k \cdot \omega\right)$,

где

$$
\begin{gathered}
\text { Gen }=v_{t} \cdot\left\{\left(\frac{\partial u_{r}}{\partial z}+\frac{\partial u_{z}}{\partial r}\right)^{2}+2 \cdot\left[\left(\frac{\partial u_{r}}{\partial r}\right)^{2}+\left(\frac{u}{r}\right)^{2}+\left(\frac{\partial u_{z}}{\partial z}\right)^{2}\right]\right\} \\
\sigma=0,5 ; C_{\mu}=0,09 ; \\
C_{1}=\frac{C_{2}}{C_{\mu}}-\frac{\sigma \cdot \chi^{2}}{\sqrt{C_{\mu}}} C_{2}=\frac{5}{6} \cdot C_{\mu} ; \chi=0,4 ;
\end{gathered}
$$

В уравнениях (1-4) использованы следующие константы и их обозначения:

- $\Omega$ - завихрённость;

- $\Psi$ - функция тока;

- Vt - коэфффициент турбулентной вязкости;

- $\sigma, \mathrm{C}_{\mu}, \mathrm{C}_{1}, \mathrm{C}_{2}$ - модельные константы.

Систему дифференциальных уравнений (1-4) решали численно методом конечных разностей. Получаемые конечно-разностные аналоги приводили к итерационной формуле Либмана, которые решали с применением метода подгонки. Для получения монотонной сходимости к решению использовали последовательную нижнюю релаксацию для всех искомых функций, кроме функции тока. Для функции тока использовали верхнюю релаксацию, что повышает скорость сходимости к решению.

В качестве граничных условий на твёрдых поверхностях использовали условие прилипания и непроникновения смеси через поверхность. Значение удельной скорости диссипации, $\omega_{w}$, на стенке определяли из условия

$$
\omega_{w}=\frac{6}{\operatorname{Re} \cdot C_{2} \cdot r^{2}}
$$

Для завихрённости $\Omega_{\mathrm{w}}$ на стенках применяли условие Тома [13], которое для нашего случая можно записать в виде

$$
\Omega_{w}=\frac{2 \cdot\left(\psi_{w-1}-\psi_{w}\right)}{(r \cdot \Delta r)^{2}},
$$

где $\psi_{w}, \psi_{w-1}-$ значения фрункций тока на стенке и в соседнем со стенкой узле разностной сетки, соответственно.

В выходном сечении рассматриваемой области использовали мягкое условие установления $\partial f / \partial z=0$, записываемое для всех зависимых переменных. На входной границе задавали равномерные или постоянные значения искомых переменных.

Поле температуры в рассматриваемой области определяли с использованием уравнения переноса теплоты, которое в безразмерном виде для избыточной температуры $\Theta=\left(T-T_{\text {хол }}\right) /\left(T_{\text {телл }}-T_{\text {хол }}\right)$, где $T, T_{\text {телл }}, T_{\text {хол }}-$ температура текущая, тёплой и холодной стенки, соответственно, можно записать в виде

$$
\begin{aligned}
& \operatorname{Re} \cdot \operatorname{Pr} \cdot r \cdot\left[u_{r} \cdot \frac{\partial \Theta}{\partial r}+u_{z} \cdot \frac{\partial \Theta}{\partial z}\right]= \\
& \frac{\partial}{\partial r}\left[r \cdot\left(1+v_{t} \cdot \frac{\operatorname{Pr}}{\operatorname{Pr}_{t}}\right) \frac{\partial \Theta}{\partial r}\right]+\frac{\partial}{\partial z}\left[r \cdot\left(1+v_{t} \cdot \frac{\operatorname{Pr}}{\operatorname{Pr}_{t}}\right) \frac{\partial \Theta}{\partial z}\right]
\end{aligned}
$$

где $\mathrm{Pr}$ - критерий Прандтля;

$\operatorname{Pr}_{t}=0,7-1,0$.

Численную реализацию программы построили на языке Pascal, графическое оформление результатов расчётов осуществляли с использованием пакета Surfer32.

Расчёт по разработанной программе проводили для десублимации высокообогащённого гексафторида урана из ПГС со следующими исходными данными:

- расход ПГС - 4,0 кг/ч;

- производительность по ГФУ - 3,0 кг/ч;

- температура тёплой стенки - $60^{\circ} \mathrm{C}$; $90^{\circ} \mathrm{C}$;

- температура холодной стенки - минус

- наружный диаметр холодной стенки, Do -320 мм; $400 \mathrm{~mm}$

- внутренний диаметр тёплой стенки, D -

Результаты решения системы уравнений (1-4) графически представлены на рисунках 4-6.

На рисунке 4 показано распределение ПОЛЗУНОВСКИЙ ВЕСТНИК № 42018 
линий тока ПГС в камере с постоянной длиной камеры $L=0,25 \cdot D_{0}$ и переменным зазором $\delta: \delta=0,02 \cdot D_{0}($ рисунок $4, a), \delta=0,04 \cdot D_{0}$ (рисунок 4, б), $\delta=0,05 \cdot D_{0}$ (рисунок 4, в).
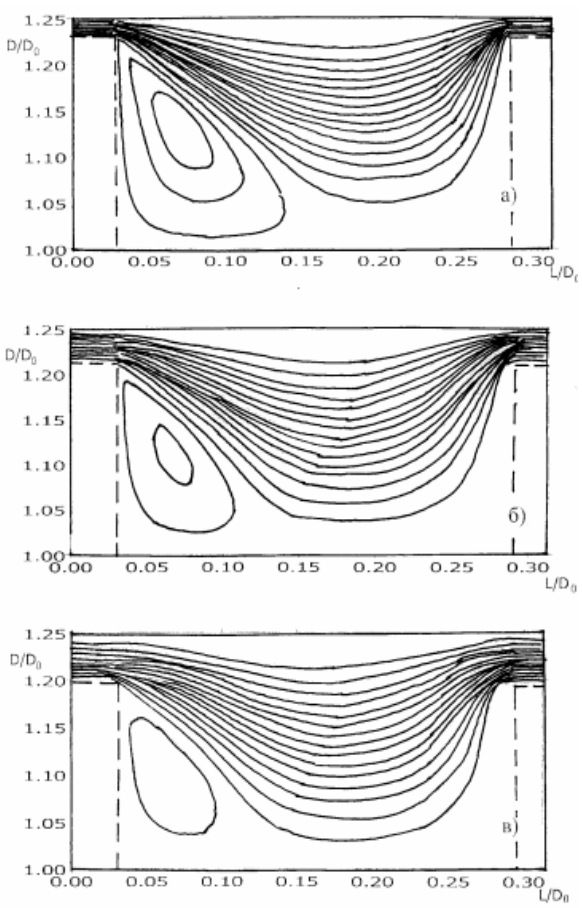

$a-\delta=0,02 \cdot D_{0} ; \sigma-\delta=0,04 \cdot D_{0}$; $\theta-\delta=0,05 \cdot D_{0}$

Рисунок 4 - Линии тока ПГС в камере при постоянной длине камеры $L$ и переменном зазоре $\delta$

Из рисунка 4 следует, что при увеличении зазора $\delta$ течение ПГС смещается к периферии аппарата, т. е. к тёплой стенке. При этом степень десублимации ГФУ в камерах снижается, а фронт конденсации растягивается по длине десублиматора. Поэтому величина этого зазора должна быть минимальной

На рисунке 5 показано распределение линий тока ПГС в камере с постоянным зазором $\delta=0,04 \cdot D_{0}$ при переменной длине камеры $L: L=0,125 \cdot D_{0}$ (рисунок $\left.5, a\right), L=0,25 \cdot D_{0}$ (рисунок $5, \sigma), L=0,375 \cdot D_{0}$ (рисунок 5, в).

Из рисунка 5 следует, что при увеличении длины камеры увеличивается глубина проникновения ПГС в камеру в направлении к холодной стенке и при $L>0,375 \cdot D_{0}$ течение ПГС выравнивается. Поэтом эту длину камеры можно принять как оптимальную.

Рассматривая распределение температуры ПГС в камере при оптимальных соотношениях геометрических параметров камеры $\delta / D_{0}$ и L/Do (рисунок 6), видно, что поле температуры в камере в этих условиях $(\delta=$ $\left.0,02 \cdot D_{0}, L=0,25 \cdot D_{0}\right)$ в достаточной степени выровнено и условия десублимации стабилизированы (рисунок 6, а). Увеличение зазора $\delta$ до $0,04 \cdot D_{0}$ (рисунок 6 , б) приводит к неравномерности поля температуры.

На рисунке 6 на линиях распределения температур цифрами обозначены температуры: 0,2 - минус $60^{\circ} \mathrm{C} ; 0,4-$ минус $30^{\circ} \mathrm{C} ; 0,6-$ $0{ }^{\circ} \mathrm{C} ; 0,8-30{ }^{\circ} \mathrm{C}$. Линии без обозначений, расположенные рядом, показывают отличие температуры на 7,5 градусов.

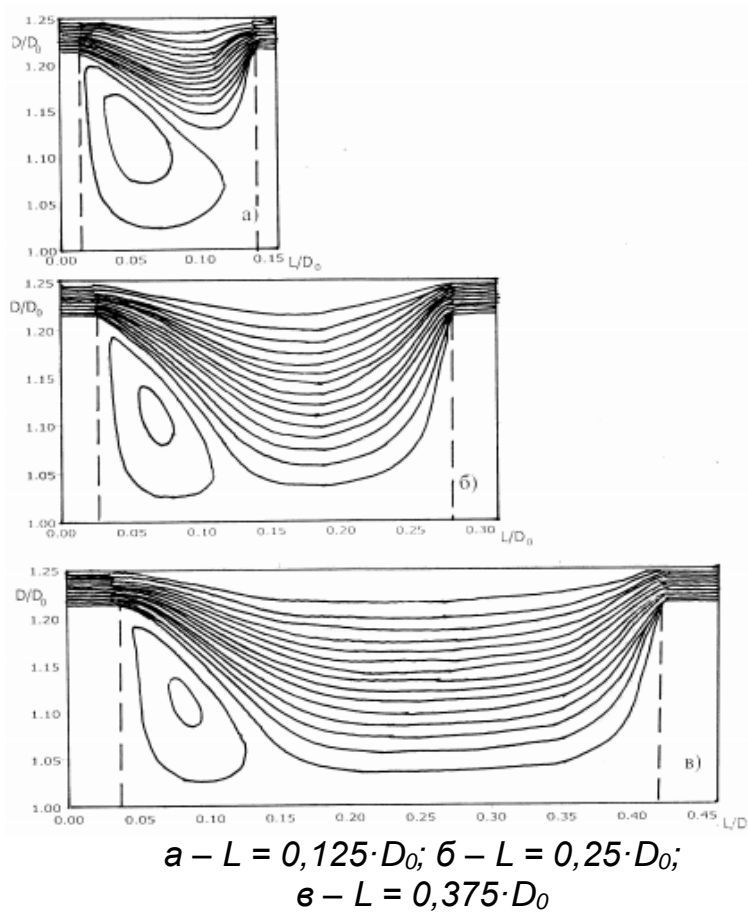

Рисунок 5 - Линии тока ПГС в камере при постоянном зазоре $\delta$ и переменной длине камеры $L$
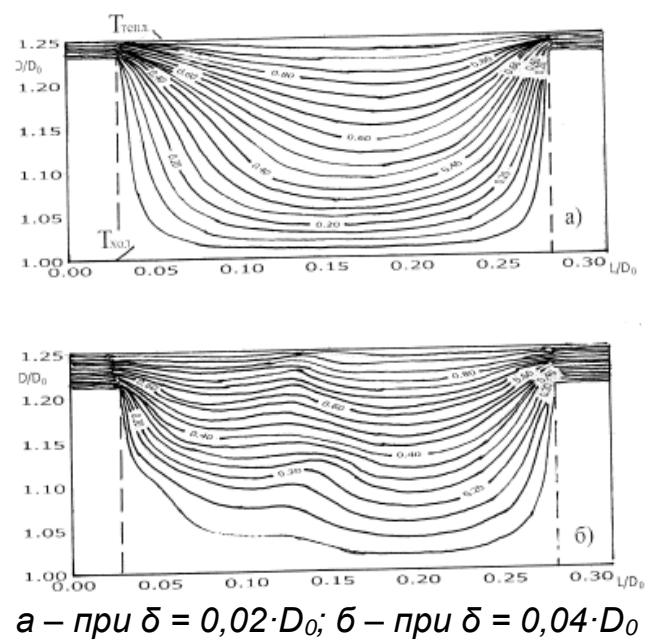

Рисунок 6 - Линии распределения температур в камере при $L=0,25 \cdot D_{0}$ 


\section{ЗАКЛЮЧЕНИЕ}

Проведены изыскания по разработке конструкции десублиматора для конденсации высокообогащённого гексафрторида урана из парогазовой смеси. Предложена конструкция многокамерного десублиматора с «тёплой» стенкой соответствующая исходным требованиям: исключение уноса аэрозолей ГФУ при контролируемой заполняемости аппарата. Разработана математическая модель десублимационного процесса применительно к многокамерному десублиматору, которая позволяет рассчитывать оптимальные параметры аппарата. Применительно к десублимации ГФУ получены графические изображения линий тока ПГС и полей температур в десублимационной камере аппарата в зависимости от её геометрических соотношений внутренних элементов.

Десублиматор соответствует правилам ядерной безопасности, конструкция аппарата защищена патентом РФ.

\section{СПИСОК ЛИТЕРАТУРЫ}

1. Шумский, К.П. Вакуумные аппараты и приборы химического машиностроения / К.П. Шумский. - М.: Машиностроение, 1974. - 576 с.

2. Горелик, А.Г. Десублимация в химической промышленности / А.Г. Горелик, А.В. Амитин. - М.: Химия, 1986. - 272 с. (Процессы и аппараты в химической и нефтехимической технологии).

3. Амелин, А.Г. Теоретические основы образования тумана при конденсации пара / А.Г. Амелин. - М.: Химия, 1972. -304 с.

4. Русаков, И.Ю. Сублимационнодесублимационные процессы для фрторидных технологий и их аппаратурное оформление : монография / И.Ю. Русаков, А.С. Буйновский, В.Л. Софронов. - Северск: Изд-во СТИ НИЯУ МИФИ. 2015. $-230 \mathrm{c}$.

5. SU 551033, МПК B01D 7/02, F28D11/06. Сублимационный конденсатор / О.Г. Молостов (СССР). - Заявл. 09.11.71, опубл. 25.05.77. Бюл. №11.

6. SU 1057058, МПК B01D 7/02. Десублиматор / В.А. Барков (СССР). - Заявл. 07.07.1981, опубл. 30.11.83. Бюл. №44.

7 RU 2271849, МПК B01D 7/02. Десублиматор / Г.И. Гриневич, В.П. Прохоров, М.Г. Макаренко $(\mathrm{RU})$. - Заявл. 08.07.2004, опубл. 20.03.2006. Бюл. №8.
8. RU 2244582, МПК В01D 7/02, B01D 59/08. Сублимационный аппарат / И.Ю. Русаков, А.А. Гущин, В.В. Лазарчук, В.А. Хохлов, Б.П. Белозёров. - заявл. 26.03.01 ; опубл. 20.02.03, Бюл. № 2.

9. Райст, П. Аэрозоли. Введение в теорию [пер. с англ.] / П. Райст. - М.: Мир, 1987. - 280 с.

10. Лойцянский, Л.Г. Механика жидкости и газа / Л.Г. Лойцянский. - изд. 6-е. - М.: Наука, 1987. $824 \mathrm{c}$.

11. Методы расчёта турбулентных течений : сборник [пер. с англ. В. И. Пономарева, Л. К. Эрдмана / под ред. В. Колльман] / Дж. Ламли, Ж. Матье, Д. Жандель [и др.]. - М.: Мир, 1984. - 463 с.

12. Уилкокс, Д.К. Уточнение уравнения для масштаба турбулентности в перспективных моделях турбулентности / Д.К. Уилкокс // Аэрокосмическая техника. - 1989. - № 11. - С. 30-46.

13. Том, А. Числовые расчёты полей в технике и физике : [пер. с англ.] / А. Том, С.Д. Эйплт. М-Л.: Энергия, 1964. - 208 с.

Русаков Игорь Юрьевич - к.т.н., доцент кафредры Машины и аппараты химических и ядерных производств Северского технологического института - филиала федерального государственного автономного образовательного учреждения высшего образования «Национальный исследовательский ядерный университет «МИФИ» (СТИ НИЯУ МИФИ), г. Северск, тел.: 8-(3823)780-173, IYRusakov@mephi.ru.

Софронов Владимир Леонидович д.т.н., профрессор кафредры Химия и технология материалов современной энергетики Северского технологического института филиала федерального государственного автономного образовательного учреждения высшего образования «Национальный исследовательский ядерный университет «МИФИ» (СТИ НИЯУ МИФИ), г. Северск, тел.: 8-(3823)780-218, VLSofronov@mephi.ru.

Брендаков Владимир Николаевич д. фриз.-мат.н., заведующий кафредрой Высшая математика и информационные технологии Северского технологического института - филиала федерального государственного автономного образовательного учреждения высшего образования «Национальный исследовательский ядерный универсuтет «МИФИ» (СТИ НИЯУ МИФИ), г. Северск, тел.: 8-(3823)780-188, e-mail: VNBrendakov@mephi.ru. 